\title{
For a greener future of ophthalmology
}

\author{
Giuseppe Giannaccare $\mathbb{C}^{1} \cdot$ João Barbosa Breda $\mathbb{1}^{2,3,4}$
}

Received: 9 December 2020 / Revised: 16 December 2020 / Accepted: 27 January 2021 / Published online: 19 February 2021

(c) The Author(s), under exclusive licence to The Royal College of Ophthalmologists 2021

\section{To the Editor:}

The modern practice of ophthalmology excels not only at reaching extraordinary outcomes but also at producing an unbelievable amount of waste on a daily basis. Cataract surgery is one of the most commonly performed surgical procedures, with an increasing surgical rate worldwide. However, its contribution to harming the environment is significant, considering that a single procedure is equivalent to the energy consumed by the average person during a week [1]. Another major source of waste in ophthalmology is related to the diffuse use of eye drop vials. Dry eye disease and glaucoma represent two highly-frequent chronic ocular disorders affecting a significant proportion of the world population $[2,3]$. Their treatment often requires the life-long use of eye drops, which in more severe cases can mean multiple products instilled several times per day. This prolonged and/or intensive use can cause toxic effects on the ocular surface, especially if preservatives are present in the product. Therefore, there has been a trend over the last decade to move towards preservative-free formulations, which can be dispensed by either unidose or multidose vials. The latter option seems to be more environmentally sustainable: there is no product waste because it can be used until the last drop; furthermore, it uses around eight times less plastic and nine times less energy for transportation. Nonetheless, there are technological challenges to producing multidose

Giuseppe Giannaccare

giuseppe.giannaccare@gmail.com

1 Department of Ophthalmology, University Magna Græcia of Catanzaro, Catanzaro, Italy

2 Cardiovascular R\&D Center, Faculty of Medicine of the University of Porto, Porto, Portugal

3 Department of Ophthalmology, Centro Hospitalar e Universitário São João, Porto, Portugal

4 Department of Neurosciences, Research Group Ophthalmology, KU Leuven, Leuven, Belgium preservative-free bottles that are both contamination-proof and cost-effective. As such, many preservative-free eyedrops are only available in unidose form.

For a greener future of ophthalmology, it would be better to move from eye drops to novel safe sustained-release drug delivery systems. In parallel, the ophthalmic community should look towards successful cases of waste reduction, such as the Aravind Eye Care System, which has shown that cutting waste is possible while maintaining high quality standards in ophthalmic surgery [4].

The current COVID-19 pandemic could temporarily slow down the greening process of ophthalmology, as both unidose systems and disposable surgical instruments should be preferred to reduce the likelihood of cross-contamination between patients and healthcare professionals.

In the future, it is desirable that environmental sustainability is considered as a core value in ophthalmology, either by adjusting current protocols to reduce waste or introducing innovative models of care built with this concept from the start [5].

Author contributions GG and JBB: conception of the work; drafting of the manuscript; critical revision of the paper.

\section{Compliance with ethical standards}

Conflict of interest The author declares no competing interests.

Publisher's note Springer Nature remains neutral with regard to jurisdictional claims in published maps and institutional affiliations.

\section{References}

1. Venkatesh R, van Landingham SW, Khodifad AM, Haripriya A, Thiel CL, Ramulu P, et al. Carbon footprint and cost-effectiveness of cataract surgery. Curr Opin Ophthalmol. 2016;27:82-8.

2. Stapleton F, Alves M, Bunya VY, Jalbert I, Lekhanont K, Malet F, et al. TFOS DEWS II epidemiology report. Ocul Surf. 2017;15: 334-65.

3. Tham YC, Li X, Wong TY, Quigley HA, Aung T, Cheng CY. Global prevalence of glaucoma and projections of glaucoma burden through 
2040: a systematic review and meta-analysis. Ophthalmology. 2014; 121:2081-90.

4. Thiel CL, Schehlein E, Ravilla T, Ravindran RD, Robin AL, Saeedi OJ, et al. Cataract surgery and environmental sustainability: waste and lifecycle assessment of phacoemulsification at a private healthcare facility. J Cataract Refract Surg. 2017;43:1391-8.

5. Klika KD. Waste plastic and pharmaceuticals, could an integrated solution help? Environ Sci Technol. 2013;47:10111-2. 